\title{
Advances in Research on the Pathogenesis of Type 2 Diabetes Complicated with Gallstone
}

\author{
Jianchu Tan ${ }^{1}$, Jiguang Kou ${ }^{2}$ \\ ${ }^{1}$ School of Medicine, Wuhan University of Science and Technology, Wuhan, China \\ ${ }^{2}$ Xiaogan Hospital, Wuhan University of Science and Technology, Xiaogan, China \\ Email: *1425514858@qq.com
}

How to cite this paper: Tan, J.C. and Kou, J.G. (2019) Advances in Research on the Pathogenesis of Type 2 Diabetes Complicated with Gallstone. International Journal of Clinical Medicine, 10, 161-173. https://doi.org/10.4236/ijcm.2019.103016

Received: February 24, 2019

Accepted: March 12, 2019

Published: March 15, 2019

Copyright () 2019 by author(s) and Scientific Research Publishing Inc. This work is licensed under the Creative Commons Attribution International License (CC BY 4.0).

http://creativecommons.org/licenses/by/4.0/

Open Access

\begin{abstract}
At present, the incidence of diabetes complicated with gallstones is increasing rapidly, and there are still many problems in the pathogenesis of the disease. Diabetes complicated with gallstones is a chronic complication of diabetes, with diabetes-induced hyperinsulinemia and insulin resistance, gallbladder emptying disorders, Oddis sphincter dysfunction, gastrointestinal hormone disorders, gastrointestinal dyskinesia, fat metabolism disorders, bile Bacterial infection and other factors are related. In recent years, it has been found that in diabetic patients, Telocytes (TC) and Cajal interstitial cells (ICC) are reduced in the biliary system. In addition, the contact between ICC cells and smooth muscle cells and nerve endings is significantly reduced, so it is considered that bile Stone formation has a certain relationship with TC and ICC reduction. This article reviews recent research progress.
\end{abstract}

\section{Keywords}

Type 2 Diabetes, Gallstones, Pathogenesis

\section{Diabetes and Gallstones}

Gallstones are stones that occur in the gallbladder, mainly cholesterol stones or mixed stones based on cholesterol. They are a common benign disease of the digestive system. The overall incidence of gallstones in Europe and the United States is between $10 \%$ and $15 \%$ [1]. The incidence of domestic reports is around $10 \%$ [2]. Diabetes mellitus (DM) is a group of metabolic diseases characterized by chronic hyperglycemia caused by multiple causes, caused by defects in insulin secretion and/or action. Acute severe metabolic disorders, such as secondary ketoacidosis, hyperosmolar hyperglycemia, can occur when the condition is severe or stressful. Studies have shown that diabetes is one of the high risk factors 
for gallbladder disease, such as gallstones, gallbladder polyps, gallbladder cancer, etc. [3] [4] [5]. The incidence of gallstones is higher in diabetic patients. An autopsy and B-ultrasound survey showed that the incidence of gallstones in diabetic patients was 2 to 3 times higher than that in non-diabetics [6]. Epidemiological surveys have shown that the incidence of gallstones in DM patients is $25 \%$ to $30 \%$ and the incidence of gallstones in T2DM patients is much higher than that in type 1 diabetes [7]. Aune $\mathrm{D}$ et al. [8] identified 10 prospective studies that could be included in a meta-analysis that further supports the increased risk of gallbladder disease in diabetic patients. At present, the study believes that the formation of gallstones is a complex process involving multiple factors, but the specific mechanism of formation of gallstones is not completely clear, especially the pathogenesis of gallstones in diabetic patients is not clear. This paper reviews the recent research progress in recent years.

\section{The Pathogenesis of Type 2 Diabetes Complicated with Gallstones}

\subsection{Hyperinsulinemia and Insulin Resistance}

Insulin resistance, defined as the reduced sensitivity of the target organ acting on insulin to insulin action. The immediate cause of insulin resistance has not been fully established, but long-term hyperglycemia and hyperinsulinemia are known to cause insulin resistance in human and animal models [9] [10]. In the study of the LIRKO mouse model, the incidence of gallstones in mice was significantly increased after disrupting the intrahepatic insulin receptor in mice [11].

3-Hydroxy-3-methylglutaryl coenzyme (HMG-CoA) reductase is the rate-limiting enzyme for cholesterol synthesis, and the stability of cholesterol in the body can be maintained by regulating the activity of the enzyme. Insulin promotes the synthesis of HMG-CoA reductase, and mouse studies have shown that insulin rapidly increases the expression of hepatic HMGR by increasing the rate of transcription [12]. High insulin accelerates the rate of cholesterol synthesis in the liver and promotes the secretion of cholesterol in the liver, which increases the cholesterol saturation in the bile. Increased serum insulin levels can induce the production of low-density lipoprotein receptor (LDLR), increase its number and up-regulate its activity [13], promote the metabolism of low-density lipoprotein from the blood into the liver and cholesterol synthesis in the liver. The increase in cholesterol synthesized in the liver leads to an increase in the amount of cholesterol secreted by the liver into the bile.

Liver X receptor a(FXR) increases the sensitivity of islet beta receptors and inhibits insulin secretion. Treatment of insulin ob/ob mice with the FXR receptor agonist GW4064 reduced serum insulin levels and increased glucose tolerance [14] [15]. The liver regulates FXRa expression significantly after insulin resistance, leading to inhibition of 7a-hydroxylase activity [16]. 7a-hydroxylase is involved in the bile acid synthesis process as a rate-limiting enzyme in the bile acid conversion process. Its activity is reduced, resulting in a significant reduction in 
bile acid and bile salt synthesis in bile, while increasing the relative content of cholesterol and mucopolysaccharide in bile. The imbalance of bile salts, cholesterol and mucopolysaccharide in bile accelerates the formation of cholesterol stones. Insulin resistance leads to decreased adiponectin expression, inhibition of insulin receptor I activation and downstream phosphatidylinositol 3-kinase signaling pathway transduction, decreased expression of fatty acid transporter 1 (FATP1) mRNA, and release of large amounts of free fatty acids by fat cells The synthesis of a large amount of cholesterol lipids leads to an increase in cholesterol in the bile and an increase in the occurrence of gallstones [17].

\subsection{Leptin}

Leptin (leptin) was discovered in 1994. It is a protein composed of 167 amino acid residues secreted by fat cells. It has functions of regulating neuroendocrine function, energy balance, feeding, growth and development [18]. Numerous studies have shown that leptin can induce cytokine signaling inhibition 3 (SOCS-3), and SOCS-3 can be feedback to regulate the expression of leptin gene [19]. In patients, insulin resistance promotes the release of leptin leading to an increase in leptin levels, and the presence of high concentrations of leptin in the blood further triggers insulin resistance. The experimental results showed that the expression of SOCS-3 was increased in obese individuals, and leptin receptor transduction was inhibited in adipocytes [20]. Decomposition of triglycerides in fat cells and beta oxidation of fatty acids are inhibited, resulting in the release of large amounts of triglycerides and fatty acids into the blood to form hyperlipidemia.

Leptin receptors are distributed in the liver, and leptin can directly act on the liver to regulate lipid metabolism. Animal experiments have found that leptin can activate lipid breakdown and inhibit lipogenesis through JAK-STAT and IRS-PI3K signaling pathways, thereby reducing lipid deposition [21]. Huynh FK et al. [22] also found that lack of hepatic leptin signaling leads to liver lipid accumulation and increases of more triglyceride-rich VLDL particles. Himeno et al. [23] found that in the absence of significant differences in energy intake and body weight, plasma insulin, triglyceride, and leptin levels were slightly elevated in mice with partial defects in leptin receptors, in the liver. The triglyceride content was significantly higher than that of the wild type, suggesting that some leptin receptor defects can cause abnormalities in liver glycolipid metabolism without affecting the central regulation of feeding activity. In insulin resistance and leptin resistance, glucose is absorbed in the small intestine, while in the liver, it inhibits phosphoenolpyruvate carboxykinase and produces a large amount of ketone bodies, and aerobic oxidation is inhibited and then turned to lipid synthesis.

Leptin can cause oxidative stress to mediate inflammation, induce peripheral blood mononuclear cells to release procoagulant factors, and promote platelet aggregation in microvessels [24], causing insufficient blood supply to the gallbladder smooth muscle to cause ischemic necrosis of gallbladder smooth 
muscle, insufficient blood supply Causes vasospasm and inflammation can further lead to inflammation of the gallbladder wall, peripheral nerve cells are also damaged to varying degrees, nerve conduction is blocked, and these factors synergistically reduce the ability of the gallbladder to move. Neuropeptide Y (NPY) and neurotransmitters such as CCK stimulate the contraction of gallbladder and Oddi. When leptin is deficient or leptin resistant, the response of the gallbladder to NPY and CCK is reduced, resulting in reduced gallbladder contractility and increased gallbladder volume, Causing bile emptying disorder [25]. A clinical study found that serum leptin levels are an important factor in the decline of gallbladder motility, because high levels of serum leptin and CCK affect cell membrane receptors, producing receptor resistance, resulting in reduced smooth muscle contractility [26]. Therefore, when leptin is relatively reduced or produces leptin resistance, it will cause gallstones due to the reduction of gallbladder contractility.

\subsection{Lipid Metabolism Disorder}

Abnormal lipid metabolism caused by diabetes is a more positive risk factor in the formation of cholelithiasis. Abnormal glucose and lipid metabolism in diabetic patients leads to hyperlipidemia [27], serum triglyceride and cholesterol are significantly elevated, and high-density lipoprotein is decreased. Clinical studies have shown that ABCG5 and ABCG8 mRNA expression is significantly increased in cholesterol stones and cholesterol polyps [28]. ABCG5/G8 is mainly expressed in the liver, gallbladder and small intestine. Studies have shown that ABCG5 and ABCG8 proteins with high expression in the liver can increase cholesterol concentration and even reach supersaturation; and the Increased expression of ABCG5 and ABCG8 protein in gallbladder epithelial cells promotes the secretion of more cholesterol from epithelial cells, and cholesterol in bile remains at high levels to form cholesterol crystals and stones [29] [30]. The sharp increase in the expression of ABCG5 and ABCG8 in patients with diabetic stones leads to an increase in cholesterol in bile. In patients with hyperlipidemia, ABCG5/G8 mRNA expression was reduced by $14 \%$ after taking atorvastatin [31].

\subsection{Bile Bacterial Infection}

Diabetic microangiopathy and autonomic neuropathy can reduce gallbladder blood supply, gallbladder emptying and reduce the body's resistance to diabetes, providing conditions for gallbladder and biliary system infections, increasing the risk of stone formation. In recent years, many scholars at home and abroad have verified the existence and role of bacteria in cholesterol gallstones through experiments. Fatemi et al. [32] found a link between Helicobacter pylori and acute gallstones. Yang Yulong et al. [33] observed 15 cases of cholesterol stones by electron microscopy, and 10 of them found bacteria in the surface structure of stones. In the transmission electron microscopy of 12 cases of cholesterol stones, 
it was found that there were 3 cases of cholesterol crystals and 12 cases of stone cores were found to have the presence of bacterial-like structural substances. The results of Tian Zhijie et al. [34] showed that Helicobacter DNA could be detected in $38.24 \%$ of bile and $79.55 \%$ of gallbladder mucosa, and 2 core parts of cholesterol mixed stones. Japanese scholar Kawai et al. [35] also detected bacterial DNA from $57 \%$ of pure cholesterol stones (100\% cholesterol). However, Demir $\mathrm{M}$ et al. [36] found no significant difference in the prevalence of Helicobacter pylori infection between diabetic patients and non-diabetic controls. It was also found that the incidence of neuropathy in diabetic patients with Helicobacter pylori infection was higher.

The mechanism by which bacteria act may be that B-glucuronidase, phospholipase, and bile acid hydrolase produced by bacteria can catalyze the hydrolysis of bile lipid components and provide a raw material for the formation of calcium precipitates. Moreover, the bacterial metabolite itself is a good substrate for the formation of crystals of cholesterol and accumulation. In addition, bacterial infection can stimulate changes in the body's immune or metabolic state, leading to gallbladder mucosal secretion disorders, gallbladder motor dysfunction, cholestasis. Therefore, some scholars have suggested that bacterial-derived substances may directly participate in the formation of cholesterol stones, and have a cross-effect with non-bacterial stone-forming mechanisms, indirectly affecting the formation of cholesterol stones by affecting or changing human immune metabolites [37]. Zhu Leiming et al. [38] found that there was a statistically significant difference in mucosal IgA and IgG between the patients with positive and negative bacterial DNA in the gallbladder mucosa $(\mathrm{P}<0.5)$, but no difference in the non-stone group $(\mathrm{P}=0.589,0.711)$. It is speculated that bacteria may cause the secretion of immunoglobulins in the gallbladder mucosa and indirectly participate in the formation of bacterial stones.

\subsection{Gallbladder Emptying Obstacles}

The gallbladder has the function of storing and enriching bile, and also has the ability to absorb part of the lipid, which depends on the relative concentration of phospholipids and cholesterol. In the case of changes in bile composition, the ability of gallbladder epithelial cells to absorb cholesterol decreases, and the concentration of cholesterol in the bile increases with the absorption of water [39]. Gallbladder contraction under physiological conditions is accomplished by hormones and neuromodulation, and changes in gallbladder dynamics can also affect the formation of gallstones. Patients with long-term fasting and complete parenteral nutrition are prone to gallstones, suggesting an important role for gallbladder contraction in the formation of stones. Ultrasound studies have shown that the fasting volume of the gallbladder and the postprandial residual volume of the gallbladder are positively correlated with obesity and high insulin [40] [41]. Cholecystokinin (CCK) is the main hormone regulating the contraction of gallbladder. CCK receptor exists on the smooth muscle of gallbladder. 
The specific binding of the two promotes the hydrolysis of phosphatidylinositol diphosphate to inositol triphosphate and diglyceride, which causes intracellular $\mathrm{Ca}^{2+}$. Induction of gallbladder contraction. Animal experiments have confirmed that the ability of the CCK gene or CCK receptor gene-deficient mice to shrink the gallbladder is significantly weakened, and the incidence of gallstones is significantly higher than that of wild-type mice [42] [43]. Studies of patients with diabetes with gallstones and non-diabetic stones have found that CCK-R and IP3-R are significantly reduced in the former, leading to impaired gallbladder emptying and gallstone formation [44]. Diabetic gallbladder autonomic neuropathy breaks the balance between sympathetic and vagal nerves, and sympathetic excitability is relatively increased. Increased levels of insulin can increase the relative excitability of the sympathetic nerves, causing gallbladder relaxation. The accumulated bile increases in viscosity under the action of gallbladder concentration, and cholesterol is easily precipitated to form crystals.

\subsection{Oddis Sphincter Dysfunction}

The annular smooth muscle at the end of the common bile duct and the end of the pancreatic duct is combined with the annular smooth muscle around the ampulla of the hepatopancreas. It is called the Oddis sphincter, also known as the hepatic and pancreatic sphincter. It has the function of controlling bile and pancreatic juice discharge. Diabetic patients often have Oddis sphincter dysfunction, which is mainly characterized by an increase in Oddis sphincter tension in the fasting state. Therefore, the increase in pressure in the common bile duct causes the bile that should flow through the Oddis sphincter into the duodenum to flow into the gallbladder, causing the gallbladder to fill too fast, and cholestasis promotes the formation of gallstones. The pathogenesis may be related to the following factors: autonomic neuropathy in diabetic patients leads to sympathetic sympathetic and vagal tone imbalance, resulting in increased Oddis sphincter tone.

\subsection{Gastrointestinal Hormone Disorder}

According to its regulation of gallbladder movement, gastrointestinal hormones can be divided into hormones that promote gallbladder movement and hormones that inhibit gallbladder movement. The hormones that promote gallbladder movement mainly include cholecystokinin, gastrin, quercetin and gastrin release. Peptides, motilin, substance $P$, etc.: The hormones that inhibit gallbladder movement mainly include vasoactive intestinal peptide, somatostatin and pancreatic polypeptide family. In diabetes, when the hormones that inhibit gallbladder movement are stronger than the hormones that promote gallbladder movement, gallstones are more likely to form [45] [46].

\subsection{Gastrointestinal Dyskinesia}

The biliary tract is closely related to gastrointestinal motility. The gallbladder 
contraction and the movement of the Oddis sphincter during the digestive phase are consistent with gastrointestinal motility. Changes in intestinal motor function also affect the contractile function of the gallbladder. Studies have shown that gastrointestinal movements are closely related to the emptying movement of the gallbladder. Gastroduodenal emptying during digestive and digestive periods can increase the emptying of the gallbladder. Emptying of the gallbladder promotes emptying of the duodenum, which in turn promotes gastric emptying, while gastrointestinal motility disorders can cause gallbladder emptying disorders. Diabetes is often accompanied by low gastrointestinal motility [47], such as stomach cramps, stomach retention, etc., which can cause gallbladder emptying disorders. Compared with the no-stone control group, the large intestine transit time of patients with gallbladder cholesterol stones, the 7- $\alpha$ dehydroxylation ability of bile acids, the amount of anaerobic bacteria, and the $\mathrm{pH}$ value of intestinal lumen were significantly increased in the intestinal flora [48]. These factors lead to a significant increase in deoxycholic acid that is returned to the bile through the enterohepatic circulation, which increases the hydrophobicity of the bile salts, thereby promoting the precipitation of bile cholesterol, increasing the cholesterol saturation index and accelerating the rate of cholesterol crystallization [49].

\subsection{Gallbladder Nerve Damage}

There are two special cell types in the gallbladder, Telocytes (TC) and Cajal interstitial cells (ICC). The term telocyte (TC) was first introduced in the scientific literature in 2010 [50]. And cells with TC characteristics have been found in almost all mammalian organs [51]. More than a century ago, Santiago Ramon and Cajal [52] described a specific cell type in the gastrointestinal tract (GI) that is located in the interstitial space between nerve endings and smooth muscle cells (SMC) and was eventually named Interstitial cells of Cajal (ICC). However, TC is related to ICC, they have the same embryonic origin (mesenchymal); both form networks that sometimes run the same region in parallel, sometimes some ICCs are embedded in the TC network, and vice versa; this strict relationship indicates TC ICC signals can be transmitted, and TC can represent ICC stem cells [51].

Matyja A et al. [53] conducted a controlled study in which bile components may play an important role in the reduction of TC density in the gallbladder. In addition, some scholars believe that TC damage may be associated with blocking c-kit/SCF signaling pathway leading to high cholesterol levels [54] or chronic inflammation of the gallbladder wall [55]. Cholesterol saturation index (CSI) is a well-established parameter associated with bile stone. Pasternak et al. [56] compared the lipid content of bile samples from patients with cholelithiasis. The results showed that the CSI of the cholelithiasis group was statistically significant and negatively correlated with the reduction of TC in the gallbladder wall. Cholesterol accumulation in gallbladder smooth muscle cells disrupts protein G-mediated signal transduction by CCK-A (cholecystokinin A) binding to its 
receptor. In another study, Pasternak et al. [54] further evaluated the relationship between bile lipid composition and TC density in the gallbladder wall of patients with gallstones and found that the concentration of omega- 6 polyunsaturated fatty acids (PUFA) was significantly increased. The average concentration of glycocholic acid (GCA) and taurocholic acid (TCA) in bile was significantly reduced. High levels of omega- 6 polyunsaturated fatty acids are shown to contribute to the formation of cholelithiasis by enhancing bile cholesterol secretion [57], while GCA and TCA may be based on a significant positive correlation with the mean number of TCs and GCA concentrations on TC Protective effects. Therefore, these observations indicate that higher concentrations of omega- 6 polyunsaturated fatty acids and lower amounts of GCA and TCA further induce gallbladder motor dysfunction and thus gallstone formation by increasing the SHI loss index caused by TC loss.

ICC has pacing and signal transduction functions in the gastrointestinal tract, which is essential for the normal operation of gastrointestinal motility. Intestinal irregularities caused by abnormal slow wave activity have been described [58] [59] [60]. ICC defects are characterized by slow wave activity abnormalities [61], and are associated with many dysmotility diseases such as slow transit constipation, hypertrophic pyloric stenosis, and pseudo intestinal obstruction. In recent years, studies have also found that ICC exists in many parts of the biliary system Oddi sphincter, cystic duct, gallbladder, etc., and participates in the regulation of Oddis sphincter autonomous rhythmic movement [62]. In human and animal models, the major cellular defect in diabetic gastroparesis is indeed the loss of ICC, which has been reported [63] [64]. In diabetes, ICC is reduced in the gallbladder and bile ducts, and in addition, ICC cells are significantly less exposed to smooth muscle cells and nerve endings [65].

\section{Conclusion and Prospects}

In short, diabetes complicated with gallstones is the result of a combination of factors, including lipid metabolism disorders, hypersaturation of cholesterol in the bile, reduction of gallbladder nerves, impaired gallbladder movement, etc., multiple factors affect each other and promote each other, resulting in a common cause Stone formation. Patients with long duration of diabetes, poor glycemic control, and elderly patients with diabetes should be alert to the formation of gallstones. It is recommended to have an abdominal ultrasound every year for early detection and treatment. Diabetes complicated with gallstones is a common chronic complication of diabetes. At present, its pathogenesis is still not fully understood. It is necessary to conduct a more in-depth study on its pathogenesis, and provide a solution for further prevention and treatment of diabetes complicated with gallstones.

\section{Conflicts of Interest}

The authors declare no conflicts of interest regarding the publication of this pa- 
per.

\section{References}

[1] Stinton, L.M. and Shaffer, E.A. (2012) Epidemiology of Gallbladder Disease: Cholelithiasis and Cancer. Gut Liver, 6, 172-187. https://doi.org/10.5009/gnl.2012.6.2.172

[2] Tan, Y.J. and Liu, Y.M. (2009) Current Status and Progress of Research on the Causes of Gallstones. Chinese Journal of Practical Surgery, 7, 602-603.

[3] Toosi, F.S., Ehsanbakhsh, A.R. and Tavakoll, M.R. (2011) Asymptomatic Gallstones and Related Risk Factors in Iran. Hepatogastroenterology, 58, 1123-1126. https://doi.org/10.5754/hge11060

[4] Cojocaru, C. and Pandele, G.I. (2010) Clinical and Paraclinical Features in Diabetic Patients Cholecystectomized for Gallstones. Revista Medico-Chirurgical a Societatii de Medici si Naturalisti din Iasi, 114, 998-1004.

[5] Unisa, S., Jagannath, P., Dhir, V., et al. (2011) Population-Based Study to Estimate Prevalence and Determine Risk Factors of Gallbladder Diseases in the Rural Gangetic Basin of North India. HPB (Oxford), 13, 117-125.

[6] Fraqudli, M., I'agliarulo, M., Colucci, A., et al. (2003) Gallbladder Motility in Obesity, Diabetes Mellitus and Coeliac Disease. Digestive and Liver Disease, 35, S12-S16. https://doi.org/10.1016/S1590-8658(03)00087-2

[7] Wang, C.C., Zhang, W.X. and Cao, Y.M. (2015) Relationship between Gallstones and Diabetes Mellitus. Journal of Hebei Medical University, 11, 1362-1364.

[8] Aune, D. and Vatten, L.J. (2016) Diabetes Mellitus and the Risk of Gallbladder Disease: A Systematic Review and Meta-Analysis of Prospective Studies. Diabetes Complications, 30, 368-373. https://doi.org/10.1016/j.jdiacomp.2015.11.012

[9] Pandey, G., Makhija, E., George, N., et al. (2015) Insulin Regulates Nitric Oxide Production in the Kidney Collecting Duct Cells. Biological Chemistry, 290, 55825591. https://doi.org/10.1074/jbc.M114.592741

[10] Martínez-Hervás, S., Vinué, A., Núñez, L., et al. (2014) Insulin Resistance Aggravates Atherosclerosis by Reducing Vascular Smooth Muscle Cell Survival and Increasing CX3CL1/CX3CR1 Axis. Cardiovascular Research, 103, 324-336. https://doi.org/10.1093/cvr/cvu115

[11] Wang, T.Y., Portincasa, P., Liu, M., et al. (2018) Mouse Models of Gallstone Disease. Current Opinion in Gastroenterology, 34, 59-70. https://doi.org/10.1097/MOG.0000000000000417

[12] Ness, G.C., Edelman, J.L. and Brooks, P.A. (2012) Involvement of Tristetraprolin in Transcriptional Activation of Hepatic 3-Hydroxy-3-Methyl Glutaryl Coenzyme A Reductase by Insulin. Biochemical and Biophysical Research Communications, 420, 178-182. https://doi.org/10.1016/j.bbrc.2012.02.138

[13] Ramakrishnan, G., Arjuman, A., Suneja, S., et al. (2012) The Association between Insulin and Low-Density Lipoprotein Receptors. Diabetes and Vascular Disease Research, 9, 196-204. https://doi.org/10.1177/1479164111430243

[14] Dufer, M., Horth, K., Krippeit-Drews, P., et al. (2012) The Significance of the Nuclear Farnesoid X receptor (FXR) in Beta cell Function. Islets, 4, 333-338. https://doi.org/10.4161/isl.22383

[15] Noel, O.F., Still, C.D., Argyropoulos, G., et al. (2016) Bile Acids, FXR, and Metabolic Effects of Bariatric Surgery. Journal of Obesity, 2016, 4390254.

[16] Fang, S., Suh, J.M., Reilly, S.M., et al. (2015) Intestinal FXR Agonism Promotes Adipose Tissue Browning and Reduces Obesity and Insulin Resistance. Nature 
Medicine, 21, 159-165. https://doi.org/10.1038/nm.3760

[17] lopez-jaramillo, P., Gomez-arbelaez, D., Lopez-lopez, J., et al. (2014) The Role of Leptin/Adiponectin Ratio in Metabolic Syndrome and Diabetes. Hormone Molecular Biology and Clinical Investigation, 18, 37-45.

[18] Zhang, Y., Proenca, R., Maffei, M., et al. (1994) Positional Cloning of the Mouse Obese Gene and Its Human Homologue. Nature, 372, 425-432. https://doi.org/10.1038/372425a0

[19] Denis, R.G., Bing, C., Brocklehurst, S., et al. (2004) Diurnal Changes in Hypothalamic Neuropeptide and SOCS-3 Expression: Effects of Lactation and Relationship with Serum Leptin and Food Intake. Journal of Endocrinology, 183, 173-181. https://doi.org/10.1677/joe.1.05659

[20] Liu, L., Liu, Y.L., Ren, Y.H., et al. (2012) Effects of Fat Cells on Leptin Response in Diet-Induced Obese Rats. Chinese Journal of Public Health, No. 5, 625-626.

[21] Zhang, L.H., Tan, X.Y., Wu, K., et al. (2015) Regulation and Mechanism of Leptin on Lipid Etabolism in Ovarian Follicle Cells from Yellow Catfish Pelteobagrus fulvidraco. General and Comparative Endocrinology, 222, 116-123. https://doi.org/10.1016/j.ygcen.2015.06.008

[22] Huynh, F.K., Neumann, U.H., Wang, Y., et al. (2013) A Role for Hepatic Leptin Signaling in Lipid Metabolism via Altered Very Low Density Lipoprotein Composition and Liver Lipase Activity in Mice. Hepatology, 57, 543-554.

https://doi.org/10.1002/hep.26043

[23] Himeno, K., Seike, M., Fukuchi, S., et al. (2009) Heterozygosity for Leptin Receptor (fa) Accelerates Hepatic Triglyceride Accumulation without Hyperphagia in Zucker Rats. Obesity Research \& Clinical Practice, 3, 1-52. https://doi.org/10.1016/j.orcp.2008.10.003

[24] Petrini, S., Neri, T., Lombardi, S., et al. (2016) Leptin Induces the Generation of Procoagulant, Tissue Factor Bearing Microparticles by Human Peripheral Blood Mononuclear Cells. Biochimica et Biophysica Acta, 1860, 1354-1361. https://doi.org/10.1016/j.bbagen.2016.03.029

[25] Lee, S., Kweon, O.K. and Kim, W.H. (2017) Associations between Serum Leptin Levels, Hyperlipidemia and Cholelithiasis in Dogs. PLoS ONE, 12, e0187315. https://doi.org/10.1371/journal.pone.0187315

[26] Zhou, W., Sun, S., Shen, F., et al. (2013) Effects of Serum Leptin and Cholecystokinin Levels on Gallbladder Contraction Function. Chinese Journal of Experimental Surgery, 30, 2275-2277.

[27] Lo, S.F., Chu, S.W., Muo, C.H., et al. (2014) Diabetes Mellitus and Accompanying Hyperlipidemia Are Independent Risk Factors for Adhesive Capsulitis: A Nationwide Population-Based Cohort Study (Version 2). Rheumatology International, 34, 67-74. https://doi.org/10.1007/s00296-013-2847-4

[28] Deng, L., Liang, M., Zhang, K., et al. (2015) The Role of ABCG5 and ABCG8 Genes in the Formation of Gallstone Cholesterol Gallstones and Cholesterol Polyps. Chinese Journal of Hepatology Surgery, No. 2, 125-128.

[29] Wang, F., Li, G., Gu, H.M., et al. (2013) Characterization of the Role of a Highly Conserved Sequence in ATP Binding Cassette Transporter G (ABCG) Family in ABCG1 Stability, Oligomerization, and Trafficking. Biochemistry, 52, 9497-9509. https://doi.org/10.1021/bi401285j

[30] Ghanbari-niaki, A., Zare-kookandeh, N. and Zare-kookandeh, A. (2014) ABCG5 Gene Responses to Treadmill Running with or without Administration of Pistachio atlantica in Female Rats. Iranian Journal of Basic Medical Sciences, 17, 162-171. 
[31] Tremblay, A.J., Lamarche, B., Lemelin, V., et al. (2011) Atorvstatin Increases Intestinal Expression of NPC1L1 in Hyperlipidemic Men. The Journal of Lipid Research, 52, 558-565. https://doi.org/10.1194/jlr.M011080

[32] Fatemi, S.M., Doosti, A., Shokri, D., et al. (2018) Is There a Correlation between Helicobacter pylori and Enterohepatic Helicobacter Species and Gallstone Cholecystitis? Middle East Journal of Digestive Diseases, 10, 24-30. https://doi.org/10.15171/mejdd.2017.86

[33] Yang, Y., Liu, X. and Tan, W. (2005) Bacteria in Cholesterol Stones and Their Role in the Mechanism of Stone Formation. Journal of Hepatobiliary and Pancreatic Surgery, 17, 14-16.

[34] Tian, Z., Han, T., Jiang, Z., et al. (2004) Study on Helicobacter DNA of Biliary System of Gallstone Disease. Chinese Journal of Practical Surgery, 24, 84-87.

[35] Kawai, M., Wahashi, M., Uchiyama, K., et al. (2002) Gram-Positive Cocci Are Associated with the Formation of Completely Pure Cholesterol Stones. The American Journal of Gastroenterology, 97, 2922-2923.

[36] Demir, M., Gokturk, H.S., Ozturk, N.A., et al. (2008) Helicobacter Pylori Prevalence in Diabetes Mellitus Patients with Dyspeptic Symptoms and Its Relationship to Glycemic Control and Late Complications. Digestive Diseases and Sciences, 53, 2646-2649.

[37] Swidsinski, A. and Lee, S.P. (2001) The Role of Bacteria in Gallstone Pathogenesis. Frontiers in Bioscience, 6, E93-E103.

[38] Zhu, L., Cai, D. and Lu, Y. (2003) A Comparative Study of Biliary Bacterial Infection Status and Immunoglobulin Correlation between Cholesterol Gallstone Patients and Non-Cholelithiasis Patients. Chinese Journal of Hepatobiliary Surgery, 9 , 419-422.

[39] Chen, W. and Liang, L. (2015) Cholecystectomy Recognition of Gallbladder Function. Chinese Journal of Practical Surgery, 35, 926-928.

[40] Lu, L., Yan, X. and Ma, S. (2011) 40 Cases of Gallbladder Emptying Dysfunction in Diabetic Patients. World Chinese Journal of Digestology, No. 3, 301-304.

[41] Jiang, Z., Han, T., Yi, F., et al. (2013) Improved B-Ultrasound Three-Dimensional Gallbladder Function Test and Judgment Criteria. Journal of Hepatobiliary and Pancreatic Surgery, No. 3, 229-231.

[42] Sato, N., Miyasaka, K., Suzuki, S., et al. (2003) Lack of Cholecys-Tokinin-A Receptor Enhanced Gallstone Formation: A Study in CCK-A Receptor Gene Knockout Mice. Digestive Diseases and Sciences, 48, 1944-1947. https://doi.org/10.1023/A:1026110002713

[43] Wang, H.H., Portincasa, P., Liu, M., et al. (2010) Effect of Gallbladder Hypomotility on Cholesterol Crystallization and Growth in CCK-Deficient Mice. Biochimica et Biophysica Acta, 1801, 138-146. https://doi.org/10.1016/j.bbalip.2009.10.003

[44] Zhang, Z., Tian, J., Liao, Q., et al. (2014) The Analysis of Expression of CCK and IP3 Receptors in Gallstones Patients with Type 2 Diabetes Mellitus. Hepatogastroenterology, 61, 2173-2176.

[45] Pendleton, H., Ekman, R., Olsson, R., et al. (2009) Motilin Concentrations in Relation to Gastro Intestinal Dysmotility in Diabetes Mellitus. European Journal of Internal Medicine, 20, 654-659. https://doi.org/10.1016/j.ejim.2009.05.015

[46] Chen, L., Zhang, X.F., Ku, B.Q., et al. (2012) Effects of Acupoint Injection of Autologous Blood on Symptoms and Plasma Motilin and Gastrin Levels of Diabetic Gastroparesis Patients. Acupuncture Research, 37, 229-232, 246. 
[47] Fan, J.G., Zhu, J., Li, X.J., et al. (2005) Prevalence of and Risk Factors for Fatty Liver in a General Population of Shanghai, China. Hepatology, 43, 508-514. https://doi.org/10.1016/j.jhep.2005.02.042

[48] Thomas, L.A., Veysey, M.J., Bathgate, T., et al. (2000) Mechanism for the Transit-Induced Increase in Colonic Deoxycholic Acid Formation in Cholesterol Cholelithiasis. Gastroenterology, 119, 806-815.

[49] van Erpecum, K.J. and van Berge Henegouwen, G.P. (2003) Intestinal Aspects of Cholesterol Gallstone Formation. Digestive and Liver Disease, 35, S8-S11. https://doi.org/10.1016/S1590-8658(03)00086-0

[50] Popescu, L.M. and Faussone-Pellegrini, M.S. (2010) Telocytes-A Case of Serendipity: The Winding Way from Interstitial Cells of Cajal (ICC), via Interstitial Cajal-Like Cells (ICLC) to Telocytes. Journal of Cellular and Molecular Medicine, 14, 729-740. https://doi.org/10.1111/j.1582-4934.2010.01059.x

[51] Vannucchi, M.G. and Traini, C. (2016) Interstitial Cells of Cajal and Telocytesin the Gut: Twins, Related or Simply Neighbor Cells? Biomolecular Concepts, 7, 93-102. https://doi.org/10.1515/bmc-2015-0034

[52] Ramon, Y. and Cajal, S. (1911) Histologie du Systeme Nerveux de L'Homme et des Vertebres. Volume 2, A. Maloine, Paris.

[53] Matyja, A., Gil, K., Pasternak, A., et al. (2013) Telocytes: New Insight into the Pathogenesis of Gallstone Disease. Journal of Cellular and Molecular Medicine, 17, 734-742. https://doi.org/10.1111/jcmm.12057

[54] Pasternak, A., Gugajska, J., Szura, M., et al. (2017) Biliary Polyunsaturated Fatty Acids and Telocytes in Gallstone Disease. Cell Transplant, 26, 125-133. https://doi.org/10.3727/096368916X692717

[55] Fan, Y., Wu, S., Fu, B., et al. (2015) The Role of Interstitial Cajal-Like Cells in the Formation of Cholesterol Stones in Guinea Pig Gallbladder. Hepatology International, 9, 612-620. https://doi.org/10.1007/s12072-015-9623-3

[56] Pasternak, A., Matyja, A., Gil, K., et al. (2013) Interstitial Cajal-Like Cells and Bile Lithogenicity in the Pathogenesis of Gall-Stone Disease. Polski Przeglad Chirurgiczny, 85, 311-316. https://doi.org/10.2478/pjs-2013-0046

[57] LaMorte, W.W., O’Leary, D.P., Booker, M.L., et al. (1993) Increased Dietary Fat Content Accelerates Cholesterol Gallstone Formation in the Cholesterol-Fed Prairie Dog. Hepatology, 18, 1498-1503.

[58] Angeli, T.R., O’Grady, G., Du, P., et al. (2013) Circumferential and Functional Re-Entry of in Vivo Slow-Wave Activity in the Porcine Small Intestine. Neurogastroenterology \& Motility, 25, e304-e314. https://doi.org/10.1111/nmo.12085

[59] O’Grady, G., Du, P., Paskaranandavadivel, N., et al. (2012) Rapid High-Amplitude Circumferential Slow Wave Propagation during Normal Gastric Pacemaking and Dysrhythmias. Neurogastroenterology \& Motility, 24, e299-e312. https://doi.org/10.1111/j.1365-2982.2012.01932.x

[60] Lammers, W.J. (2013) Arrhythmias in the Gut. Neurogastroenterology \& Motility, 25, 353-357. https://doi.org/10.1111/nmo.12116

[61] Farrugia, G. (2008) Interstitial Cells of Cajal in Health and Disease. Neurogastroenterology \& Motility, 20, 54-63. https://doi.org/10.1111/j.1365-2982.2008.01109.x

[62] Ahmadi, O., Nicholson Mde, L., Gould, M.L., et al. (2010) Interstitial Cells of Cajal Are Present in Human Extrahepatic Bile Ducts. Gastroenterology \& Hepatology, 25, 277-285.

[63] Grover, M., Farrugia, G., Lurken, M.S., et al. (2011) Cellular Changes in Diabetic 
and Idiopathic Gastroparesis. Gastroenterology, 140, 1575-1585e8.

[64] Choi, K.M., Gibbons, S.J., Nguyen, T.V., et al. (2008) Heme Oxygenase-1 Protects Interstitial Cells of Cajal from Oxidative Stress and Reverses Diabetic Gastroparesis. Gastroenterology, 135, 2055-2064.

[65] Faussone-Pellegrini, M.S., Grover, M., Pasricha, P.J., et al. (2012) Ultrastructural Differences between Diabetic and Idiopathic Gastroparesis. Journal of Cellular and Molecular Medicine, 16, 1573-1581.

https://doi.org/10.1111/j.1582-4934.2011.01451.x 